\title{
Routing in Accumulative Multi-hop Networks
}

\author{
Jesus Gomez-Vilardebo \\ Centre Tecnològic de Telecomunicacions de Catalunya (CTTC) \\ Castelldefels, Spain \\ jesus.gomez@cttc.es
}

\begin{abstract}
This paper investigates the problem of finding optimal paths in single-source single-destination accumulative multi-hop networks. We consider a single source that communicates to a single destination assisted by several relays through multiple-hops. At each hop, only one node transmits, while the rest of nodes receive the transmitted signal, and store it after processing/decoding and mixing with the signals received in previous hops. This is, we consider that terminals make use of advanced energy accumulation transmission/reception techniques such us maximal ratio combining reception of repetition codes, or information accumulation with rateless codes. Accumulative techniques increase communication reliability, reduce energy consumption, and decrease latency. We investigate the properties that a routing metric must satisfy in these accumulative networks to guarantee that optimal paths can be computed with Dijkstra's algorithm. We model the problem of routing in an accumulative multi-hop networks, as the problem of routing in a hypergraph. We show that optimality properties in traditional multi-hop network (monotonicity and isotonicity) are no longer valid and derive a new set of sufficient conditions for optimality.
\end{abstract}

\section{INTRODUCTION}

Introducing relay capabilities in a network has a strong effect on the information flow that extends to all communication levels, from the achievable rates to the routing strategy. A fundamental understanding of the role that relays play in wireless networks is of paramount importance to the design of efficient protocols for future communication systems.

The problem of routing in a traditional multi-hop (TM) network model, where each relay node only listens to the immediately previous node is quite well understood today. For the purpose of routing, these networks are well modeled by directed graphs. Given a routing metric criteria, the optimality conditions that guarantee that efficient path search algorithms, such as Dijkstra's algorithm, find the optimal path were studied in [1], [2].

The problem of routing in an accumulative multi-hop (AM) network model, in which we are instead interested, is however far from being understood today. In accumulative multi-hop networks, a single source communicates to a single destination assisted by several relay nodes that can accumulate the received energy/information from previous relay transmissions. In practice, there are two main accumulation mechanisms

This work was partially supported by the Catalan Government under grant SGR2014-1567, the Spanish Government under grant PCIN-2013-027 (ECROPS), and TEC2013-44591-P (INTENSYV), and the European project FP7-ICT-2011-8 NEWCOM\# 318306. at relays: energy and mutual-information accumulation. Energy accumulation can be performed at the receiving nodes, e.g., through space-time coding or repetition coding [3], [4]. Mutual-information accumulation [5], [6] can be realized using rateless codes e.g. fountain or raptor codes [7]. Accumulation mechanisms are considered in current and next generation standards since they increase communication reliability and reduce energy consumption.

The work presented here builds, mainly, on top of the works conducted in [1], [2]. We show that graphs can not model the AM network, and thus, the results derived in [1], [2] for routing over graphs can not be invoked. We model the AM network by a hypergraph, and find new sufficient conditions to guarantee the optimality of Dijkstra's algorithm in hypergraphs. We then discuss the optimality of Dijkstra's algorithm for the minimum energy routing in static AM networks. In the case of decoded-and-forward (DF) relaying this problem has been previously addressed in [3], [4], [7][10]. DF relay nodes decode the source message completely by accumulating energy, or information from all previous transmissions. From [3] and [4], we already know that finding the optimal transmission order for these networks is an NPcomplete problem. We show the optimality of Dijkstra's algorithm for DF accumulative networks where nodes decode the source message by only accumulating the energy/information coming from the immediately previous relay, and from the source. Besides the DF relaying, we also consider the cut-set bound (CB) for AM networks [11, Th. 14.10.1]. For the CB, we show that Dijkstra's algorithm finds the minimum energy route.

The remainder of the paper is organized as follows. The AM network model is presented in Section II. In Section III, the minimum energy accumulative path weight function for DF relaying and for the $\mathrm{CB}$ are derived. The optimality of Dijkstra' algorithm in AM networks is discussed in Section IV, and particularized for the minimum energy accumulative routing metrics in Section V. Finally, conclusions are drawn in Section VI.

\section{The Accumulative Network Model}

Consider a static network with $N$ nodes. The traffic is unicast, from a source node (S) to a destination node (D) with the help of relay transmissions. Relay nodes transmit according to a given transmission order described by a path vector $\mathbf{p}$, where $\mathbf{p}[0]=\mathrm{S}, \mathbf{p}[L+1]=\mathrm{D}$, and $L$ is the number of relays. Notice that we only allow one relay node in each path 


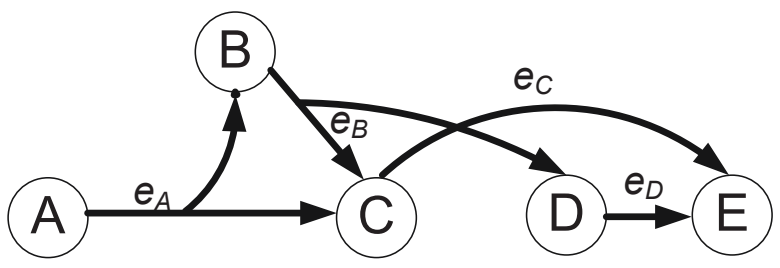

Fig. 1: A directed hypergraph $\mathcal{H}$. The arrow on a hyperedge in the figure points to the vertices in the head of the hyperedge.

position. Communications are either point-to-multipoint as in wireless channels, or point-to-point as in wireline channels.

In a TM network model, given a path $\mathbf{p}$, the signal transmitted by node $\mathbf{p}[i]$ is only intended to node $\mathbf{p}[i+1]$. This is so, even if transmissions are over wireless channels, and the transmitted signals are also overhead by nodes in the path other than the intended ones. These nodes ignore or treat as interference the received signals. In TM routing problems, the network is well modeled by a directed graph $\mathcal{G}(V, E)$, where $V$ is the set of nodes and $E$ is the set of edges representing the existence of links between pairs of nodes. Let $e_{\mathrm{u}, \mathrm{v}}$ denote the edge between nodes $\mathbf{u}$ and $\mathbf{v}$. A path $\mathbf{p}$ exits if $e_{\mathbf{p}[i], \mathbf{p}[i+1]} \in E$ for all $i=\{0, \ldots, L\}$. Associated to each edge, there can be one or several fixed metrics, e.g. the link distance, the link bandwidth, the channel magnitude, the transmission delay, etc. For simplicity, let us as assume that there is only one metric per edge, then $\beta\left(e_{\mathrm{u}, \mathrm{v}}\right)=\beta_{u, v}$ denotes the metric associated to edge $e_{\mathrm{u}, \mathrm{v}}$. In TM routing the objective is to find the better path, or lightest path, between a source and a destination according to some network metric. The weight of a path $w(\mathbf{p})$ is a function of the metrics of edges traversed by a path, namely $w(\mathbf{p})=w\left(\beta_{\mathbf{p}}\right)$, where $\beta_{\mathbf{p}}=\left\{\beta_{\mathbf{p}[i], \mathbf{p}[i+1]}, i=0, \ldots, L\right\}$.

In the AM model, in which we are instead interested, relay nodes do not discard the received signals from previous nodes in the path. This is, relay and destination nodes may benefit from the signals received from all previous nodes in the path. In AM routing, the network is better modeled by a directed hypergraph $\mathcal{H}(V, E)$, as the one shown in Figure 1 , where $V$ denotes the set of nodes, or vertices, and $E$ denotes the set of hyperedge, or connections between nodes. A directed hypergraph is a generalization of a directed graph in which each hyperedge is allowed to have multiple source (tail) vertices and multiple destination (head) vertices. The tail and head vertices of an edge are denoted as $T(e)$ and $H(e)$, respectively. We restrict the analysis to hypergraphs where all the edges have only one source node $|T(e)|=1$, and there is only one edge per source node. This hypergraph model is sufficiently general to consider any wireless communication in accumulative network if there is only one node in each path position. There are many different notions of hyperpaths, see [12]. Here, we define a hyperpath as a sequence of nodes $\mathbf{p}=[\mathbf{p}[0], \ldots, \mathbf{p}[L+1]]$ consisting of vertices $\mathbf{p}[i] \in V$. Let $e_{\mathrm{u}}$ denote the hyperedge associated to node $\mathrm{u}$. A hyperpath exists if for every node in the path $\mathbf{p}[i], 0<i \leq L+1$ there exits at least one preceding node $\mathbf{p}[j], 0 \leq j<i$, such that $\mathbf{p}[i] \in H\left(e_{\mathbf{p}[j]}\right)$. The existence condition of a hyperpath is illustrated in Fig. 1. Observe that since there is no edge link connecting nodes $\mathrm{C}$ and $\mathrm{D}$, the path $\langle\mathrm{A}, \mathrm{B}, \mathrm{C}, \mathrm{D}, \mathrm{E}\rangle$ does not exists in a graph. However, the hyperpath $\langle A, B, C, D, E\rangle$ exists, as there is an hyperedge connecting nodes $\mathrm{B}$ and $\mathrm{D}$. Associated to an hyperedge there might be a set of metrics. For simplicity, we assume that there is only one metric for each of the vertices of the hyperedge, namely, $\beta\left(e_{\mathrm{u}}\right)=\left\{\beta_{\mathrm{u}, \mathrm{v}}, \forall \mathrm{v} \in H\left(e_{\mathrm{u}}\right)\right\}$. Then, the weight of a hyperpath $w(\mathbf{p})$ is a function of the metrics of the edges traversed by the path, namely $w(\mathbf{p})=w\left(\beta_{\mathbf{p}}\right)$, where $\beta_{\mathbf{p}}=\left\{\beta\left(e_{\mathbf{p}[i]}\right), i=\{0, \ldots, L+1\}\right\}$.

\section{Minimum Energy Accumulative Routing}

The implications of accumulative nodes in multi-hop routing problems are better understood by looking at specify examples of path weight functions. The path weight functions derived here will also be instrumental in subsequent sections.

We consider a very simplistic accumulative communication model for wireless channels. The link between nodes $\mathrm{u}$ and $\mathrm{v}$ is modeled by the channel gain $g_{\mathrm{u}, \mathrm{v}} \in\left\{\mathbb{R}^{+}, 0\right\}$. Let $P_{\mathrm{u}}$ denote the transmission power at node $\mathrm{u}$, then the received signal power at node $\mathrm{v}$ is $g_{\mathrm{u}, \mathrm{v}} P_{\mathrm{u}}$. A packet is correctly decoded at the destination node if the received signal power at the destination node exceeds a certain threshold level $H_{\mathrm{D}}$. This model is valid for network that operate in the wideband power limited regime. This regime is realistic for some wireless networks, such as sensor networks where there exist strong energy limitations at nodes, the traffic load is low, and there is sufficient large frequency bandwidth. Moreover, the analysis conducted here can be extended to any other scenario where the resource allocation problem is linear. Such linear dependence is forced here by considering energy accumulation in wideband signals, as in [3], or in [4], but it can also be found in other situations, such as when considering full-duplex relay terminals as in [8], or when optimizing over transmission durations instead of transmitted power as in [7] and [13].

Our network metric is the aggregated transmission power consumption needed to successfully transmit a packet from the source to the destination node by using wireless links. Accordingly, we define the weight of the path $\mathbf{p}$ as

$$
w(\mathbf{p})=\frac{\sum_{i=0}^{L} P_{\mathbf{p}[i]}}{H_{D}} .
$$

If the above network is modelled by graph, then the metric associated to the edge $e_{\mathrm{u}, \mathrm{v}}$ is the channel gain $\beta\left(e_{\mathrm{u}, \mathrm{v}}\right)=g_{\mathrm{u}, \mathrm{v}}$. In contrast, if the network is described by a hypergraph, then the metric associated to the edge of node $u$ is the set of channel gains from node $\mathrm{u}$ to all the network nodes, namely $\beta\left(e_{\mathbf{u}}\right)=$ $\left\{g_{\mathrm{u}, \mathrm{v}}, \forall \mathrm{v}\right\}$.

For the sake of simplicity, when there is no ambiguity on which path $\mathbf{p}$ we are referring to, we denote $g_{i, j}=g_{\mathbf{p}[i], \mathbf{p}[j]}$, and $P_{i}=P_{\mathbf{p}[i]}$. 


\section{A. Decode-and-forward relaying}

Let us first suppose that relay nodes must decode the source message before transmission, and thus, they need to receive an aggregated signal power also exceeding $H_{\mathrm{D}}$. Consider a predetermined power transmission strategy, in which, node $\mathbf{p}[i-1]$ transmits the minimum power need for node $\mathbf{p}[i]$ to accumulate exactly $H_{\mathrm{D}}$ units of energy.

In a TM network, according to this communication model, the power transmitted by the source node must satisfy $H_{\mathrm{D}}=$ $g_{0,1} P_{0}$. The weight of the partial path between the source and the first relay, denoted as $p_{0,1}$, is given by

$$
w\left(\mathbf{p}_{0,1}\right)=\frac{P_{0}}{H_{\mathrm{D}}}=\frac{1}{g_{0,1}} .
$$

In the next hop, given that node $\mathbf{p}[2]$ ignores the signal power received from the source node, the power transmitted by node $\mathbf{p}$ [1] must satisfy $H_{\mathrm{D}}=g_{1,2} P_{1}$, and thus, the weight of the partial path $\mathbf{p}_{0,2}$ is given by

$$
\begin{aligned}
w\left(\mathbf{p}_{0,2}\right) & =\frac{P_{0}+P_{1}}{H}, \\
& =\frac{1}{g_{0,1}}+\frac{1}{g_{1,2}}, \\
& =\frac{1}{g_{1,2}}+w\left(\mathbf{p}_{0,1}\right) .
\end{aligned}
$$

It can be shown, that the weight of the partial path $\mathbf{p}_{0, i}$ is computed recursively as

$$
w\left(\mathbf{p}_{0, i}\right)=\frac{1}{g_{i-1, i}}+w\left(\mathbf{p}_{0, i-1}\right) .
$$

In the AM network, instead, all the nodes except the source, and the first hop relay node can get multiple energy leakages from previous transmissions, and thus accumulate energy from them. The power transmitted by the source node must also satisfy $\mathrm{H}_{\mathrm{D}}=g_{0,1} P_{0}$, and thus, $w\left(\mathbf{p}_{0,1}\right)=\frac{1}{g_{0,1}}$. However, the power transmitted by the first relay must satisfy

$$
H_{\mathrm{D}}=g_{1,2} P_{1}+g_{0,2} P_{0} .
$$

The weight of the partial path $w\left(\mathbf{p}_{0,2}\right)$ is given by

$$
\begin{aligned}
w\left(\mathbf{p}_{0,2}\right) & =\frac{P_{0}+P_{1}}{H}, \\
& =\frac{1}{g_{0,1}}+\frac{1}{g_{1,2}}\left(1-\frac{g_{0,2}}{g_{0,1}}\right), \\
& =\frac{1}{g_{1,2}}+\frac{g_{1,2}-g_{0,2}}{g_{1,2}} w\left(\mathbf{p}_{0,1}\right) .
\end{aligned}
$$

Observe that, if $g_{0,2}>g_{0,1}$, or, equivalently, if $w\left(\mathbf{p}_{0,2}\right)<$ $w\left(\mathbf{p}_{0,1}\right)$, then the relay node $\mathbf{p}[2]$ has already received sufficient power from the source node, and thus enforcing (2) implies $P_{1}<0$, which is not possible. In that case, we set $w\left(\mathbf{p}_{0,2}\right)=\infty$, as it can be shown, see [4], that if we remove node $\mathbf{p}[1]$ from the path, we obtain a smaller (better) path weight. It can be shown, that then the weight of the partial path $\mathbf{p}_{0, i}$ is computed recursively as

$$
w\left(\mathbf{p}_{0, i}\right)=\frac{1}{g_{i-1, i}}+\sum_{j=1}^{i-1} \frac{g_{j, i}-g_{j-1, i}}{g_{i-1, i}} w\left(\mathbf{p}_{0, j}\right)
$$

if $w\left(\mathbf{p}_{0, i}\right) \geq w\left(\mathbf{p}_{0, i-1}\right)$, and $w\left(\mathbf{p}_{0, i}\right)=\infty$ otherwise.

Observe that this accumulative path weight function could not be computed over a directed graph.

\section{B. Cut-set bound}

In the AM network model, asking every node to decode the source message is not always needed. Relays can, for example, amplify or compress and forward the received signals, without decoding the information. We can have an idea of the path weight functions that may appear for these non-regenerative relaying strategies by looking at the cut-set bound. In addition, recent results [14] have shown that the cut-set bound rates can be achieved within a constant rate gap by compress and forward like strategies. To model these communication, we remove the decoding constraint at nodes, and consider a power transmission policy in which the power transmitted by node $\mathbf{p}[i]$ is such that subsequent nodes in the path $\mathbf{p}[j], j>i$ receive an aggregated power equal to the power that node $\mathbf{p}[i]$ has received from previous nodes $\mathbf{p}[j], j<i$, namely

$$
P_{i} \sum_{j=i+1}^{L+1} g_{i, j}=\sum_{j=0}^{i-1} P_{j} g_{j, i} .
$$

In this case, it is more convenient to compute the weight of a path in a backward manner, from the destination to the source. Only for this case, let us reverse the ordering of nodes, so that the destination is refereed to as $\mathrm{D}=\mathbf{p}[0]$, and the source as $\mathbf{S}=\mathbf{p}[L+1]$, the channel gain from node $i$ to node $j$ is denoted as $g_{j, i}$, and the path from node $i$ to the destination is denoted as $\mathbf{p}_{0, i}=\langle\mathbf{p}[i], \ldots, \mathbf{p}[0]\rangle$. Then, the signal power received from the last relay at the destination node is given by $g_{0,1} P_{1}$, and the weight of the partial path $\mathbf{p}_{0,1}=\langle\mathbf{p}[1], \mathbf{p}[0]\rangle$ is

$$
w\left(\mathbf{p}_{0,1}\right)=\frac{P_{1}}{g_{0,1} P_{1}}=\frac{1}{g_{0,1}} .
$$

For the partial path $\mathbf{p}_{0,2}=\langle\mathbf{p}[2], \mathbf{p}[1], \mathbf{p}[0]\rangle$ the destination receives an aggregated power of $g_{0,1} P_{1}+g_{0,2} P_{2}$, accordingly, the weight of the partial path $\mathbf{p}_{0,2}$ is

$$
w\left(\mathbf{p}_{0,2}\right)=\frac{P_{1}+P_{2}}{g_{0,1} P_{1}+g_{0,2} P_{2}}
$$

by enforcing at node $\mathbf{p}[1]$, the input-output power flow condition in (4), we require $g_{1,2} P_{2}=P_{1} g_{0,1}$, obtaining

$$
w\left(\mathbf{p}_{0,2}\right)=\frac{1+g_{1,2} w\left(\mathbf{p}_{0,1}\right)}{g_{1,2}+g_{0.2}}
$$

Similarly, for the partial path $\mathbf{p}_{0,3}$, the destination receives an aggregated power of $g_{0,1} P_{1}+g_{0,2} P_{2}+g_{0,3} P_{3}$, and the inputoutput power flow condition at relay nodes $\mathbf{p}[2]$, and $\mathbf{p}[1]$, requires

$$
\begin{aligned}
g_{1,2} P_{2}+g_{1,3} P_{3} & =P_{1} g_{0,1}, \\
g_{2,3} P_{3} & =P_{2}\left(g_{0,2}+g_{1,2}\right) .
\end{aligned}
$$

The weight of the partial path $\mathbf{p}_{0,3}$ is then given by

$$
w\left(\mathbf{p}_{0,3}\right)=\frac{1+g_{1,3} w\left(\mathbf{p}_{0,1}\right)+g_{2,3} w\left(\mathbf{p}_{0,2}\right)}{g_{2,3}+g_{1,3}+g_{0,3}} .
$$


It can be shown that the weight of the partial path $\mathbf{p}_{0, i}$ is computed recursively as

$$
w\left(\mathbf{p}_{0, i}\right)=\frac{1+\sum_{j=1}^{i-1} g_{j, i} w\left(\mathbf{p}_{0, j}\right)}{\sum_{j=0}^{i-1} g_{j, i}} .
$$

Observe that we can only compute this path weight over a hypergraph.

\section{Optimality of DiJkstra's Algorithm in ACCUMULATIVE NETWORKS}

In this section, we present sufficient conditions to guarantee that Dijkstra's algorithm finds the lightest path over a directed hypergraph $\mathcal{H}(V, E)$. We begin by providing the mathematical representation of a path selection criteria which is usually called as routing metric. We represent a routing metric following the notation in [2] as an algebra on top of a quadruplet $(Q, \oplus, w, \preceq)$, where $Q$ is the set of all possible paths, $\oplus$ is a binary operation that maps pairs with a path and an ordered sequence of nodes into a path, i.e. if the path $\mathbf{a} \in Q$ and the last node in a coincides with the first node of the ordered sequence of nodes $\mathbf{b}$, then $\mathbf{a} \oplus \mathbf{b}$ denotes the concatenation of path a with the ordered sequence of nodes $\mathbf{b}$, with $\mathbf{a} \oplus \mathbf{b} \in Q$, $w$ is a function that maps a path to a weight, and $\preceq$ is an order relation, where $w(\mathbf{a}) \preceq w(\mathbf{b})$ means the path $\mathbf{a}$ is lighter (better) that or equal to $\mathbf{b}$. Given a routing metric $(Q, \oplus, w, \preceq)$, a routing protocol operates with the path weights of the paths in $Q$ to find the lightest path $\mathbf{q}^{*} \in Q$ between a source and a destination.

The concatenation operation as defined above differs slightly from the one defined in [2] for graphs. In [2], $\oplus$ concatenates two paths in $Q$, and returns a path also in $Q$. The definition of $\oplus$ presented here is motivated by the fact that in a hypergraph, even if the ordered set of nodes $\mathbf{b}$ does not belong to $Q$, the path $\mathbf{a} \oplus \mathbf{b}$ might belong to $Q$.

\section{A. Optimality Conditions in Traditional Multi-hoping}

Here we review the conditions that guarantee that Dijkstra's algorithm finds the lightest path in a directed graph $\mathcal{G}(V, E)$, and discuss their extension to directed hypergraphs $\mathcal{H}(V, E)$.

Given a graph, references [1] and [2] developed a comprehensive framework to identify the specific conditions a routing metric needs to satisfy in order to be combined with a certain type of optimal routing protocol to obtain the optimal path. In particular, it was shown that Dijkstra's algorithm with source routing is optimal if and only if, the routing metric satisfies right-monotonicity and right-isotonicity. These properties are here stated, mostly, as they appear in [2] with the necessary modifications to account for the new definition of the binary operation $\oplus$.

Definition 1: The quadruplet $(Q, \oplus, w, \preceq)$ is rightmonotonic if $w(\mathbf{a}) \preceq w(\mathbf{a} \oplus \mathbf{b})$, for any paths $\mathbf{a}, \mathbf{a} \oplus \mathbf{b}$ in $Q$.

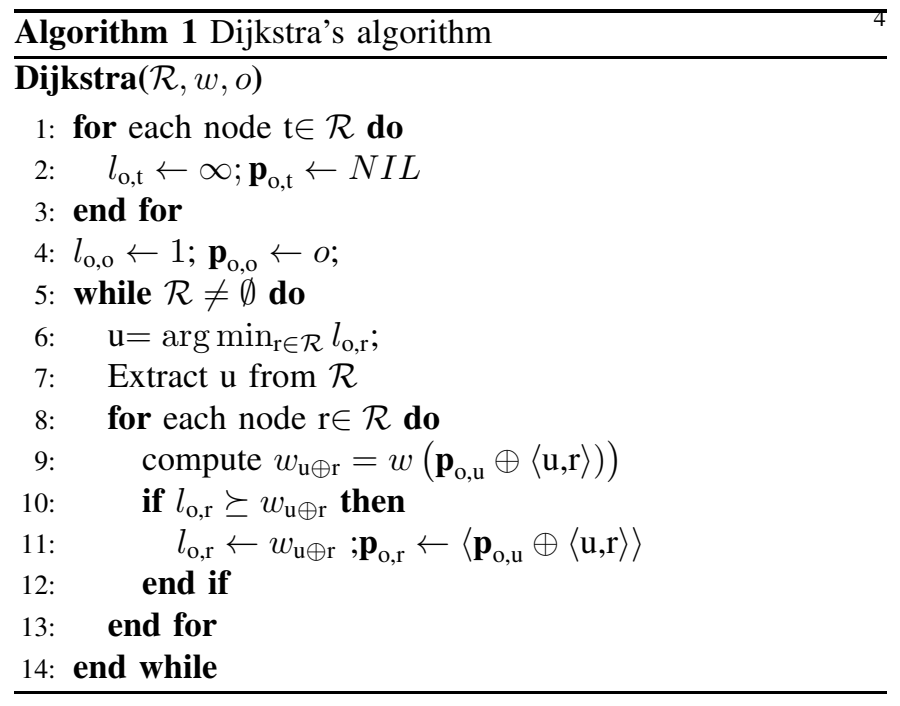

Definition 2: Given the paths $\mathbf{a}$ and $\mathbf{b}$ between two nodes $\mathrm{A}$ and $\mathrm{B}$, and the paths $\mathbf{a} \oplus \mathbf{c}$ and $\mathbf{b} \oplus \mathbf{c}$ from $\mathrm{A}$ to a third node $\mathrm{C}$, sharing the nodes in c. If $w(\mathbf{a}) \preceq w(\mathbf{b})$, the quadruplet $(Q, \oplus, w, \preceq)$ is right-isotonic if $w(\mathbf{a} \oplus \mathbf{c}) \preceq w(\mathbf{b} \oplus \mathbf{c})$ for any paths $\mathbf{a}, \mathbf{b}, \mathbf{a} \oplus \mathbf{c}$ in $Q$.

This definition of right-monotonicity differs from the one presented in [2], in that it does not restrict the paths $\mathbf{b}$ to belong to $Q$. Similarly, the definition of right-isotonicity differs form the one in [2] in that it does not restrict the paths c to those in $Q$.

If the network is modeled by a directed graph as in the TM network model, then the right-monotonicity and rightisotonicity conditions are necessary and sufficient conditions for Dijkstra's algorithm to find the lightest path. However, if the network needs to be modeled as a hypergraph, as is likely the case in AM networks, then these conditions are no longer necessary. Satisfying both conditions is still sufficient for Dijkstra's algorithm to find the lightest path. The proof of sufficiency follows exactly the one provided in [2] for graphs, and is thus not reproduced here. The lack of necessity is demonstrated next, by presenting an alternative set of sufficient conditions for the optimality of Dijkstra's algorithm.

\section{B. Optimality Conditions in Accumulative Multi-hoping}

Although right-monotonicity and right-isotonicity conditions are sufficient to show the optimality of Dijkstra's algorithm, they might not be very helpful for path weight function in AM networks. The right-isotonicity condition, for instance, can only be satisfied if there is a certain decoupling between the nodes in paths $\mathbf{a}$, or $\mathbf{b}$, and those in path $\mathbf{c}$. However, it is precisely, the connection between these nodes what we want to include by considering AM networks. In the following, we present a new set of sufficient conditions that guarantee the optimality of Dijkstra's algorithm in directed hypergraphs, with only one hyperedge per node.

Definition 3 (Condition C1): Consider a route metric $(Q, \oplus, w, \preceq)$ defined in a hypergraph with only one edge per node. Given any path a whose last node is $\mathrm{A}$, and the paths 
$\mathbf{a} \oplus\langle\mathrm{A}, \mathrm{B}\rangle$ and $\mathbf{a} \oplus\langle\mathrm{A}, \mathrm{C}\rangle$ sharing the common root path a, we say that the route metric satisfies condition $\mathrm{C} 1$ if satisfying any of the conditions below, implies satisfying all the others

$$
\begin{aligned}
w(\mathbf{a} \oplus\langle\mathrm{A}, \mathrm{B}\rangle) & \preceq w(\mathbf{a} \oplus\langle\mathrm{A}, \mathrm{C}\rangle), \\
w(\mathbf{a} \oplus\langle\mathrm{A}, \mathrm{B}\rangle) & \preceq w(\mathbf{a} \oplus\langle\mathrm{A}, \mathrm{B}, \mathrm{C}\rangle), \\
w(\mathbf{a} \oplus\langle\mathrm{A}, \mathrm{B}, \mathrm{C}\rangle) & \preceq w(\mathbf{a} \oplus\langle\mathrm{A}, \mathrm{C}\rangle), \\
w(\mathbf{a} \oplus\langle\mathrm{A}, \mathrm{B}\rangle) & \preceq w(\mathbf{a} \oplus\langle\mathrm{A}, \mathrm{C}, \mathrm{B}\rangle), \\
w(\mathbf{a} \oplus\langle\mathrm{A}, \mathrm{C}, \mathrm{B}\rangle) & \preceq w(\mathbf{a} \oplus\langle\mathrm{A}, \mathrm{C}\rangle)
\end{aligned}
$$

for any paths $\mathbf{a} \oplus\langle\mathrm{A}, \mathrm{B}\rangle, \mathbf{a} \oplus\langle\mathrm{A}, \mathrm{C}\rangle$ belonging to $Q$. Observe that for hyperpaths with only one edge per node it is guaranteed that the paths $\mathbf{a} \oplus\langle\mathrm{A}, \mathrm{B}, \mathrm{C}\rangle$, and $\mathbf{a} \oplus\langle\mathrm{A}, \mathrm{C}, \mathrm{B}\rangle$ also belong to $Q$.

Definition 4 (Condition C2): A path weight satisfies condition $\mathrm{C} 2$ if for any ordered set of nodes $\mathbf{c}$, with partial paths $\mathbf{c}_{0, j}=\langle\mathbf{c}[0], \ldots, \mathbf{c}[j]\rangle, j=0, \ldots,|\mathbf{c}|-1$ satisfying $w(\mathbf{a} \oplus\langle\mathrm{A}, \mathrm{B}\rangle)) \preceq w\left(\mathbf{a} \oplus\left\langle\mathbf{A}, \mathbf{c}_{0, j}\right\rangle\right)$ for all $j$, implies that

$$
w(\mathbf{a} \oplus\langle\mathrm{A}, \mathbf{B}, \mathbf{c}\rangle) \preceq w(\mathbf{a} \oplus\langle\mathbf{A}, \mathbf{c}\rangle)
$$

for any paths $\mathbf{a} \oplus\langle\mathrm{A}, \mathrm{B}\rangle$, and $\mathbf{a} \oplus\langle\mathrm{A}, \mathbf{c}\rangle \in Q$.

Condition $\mathrm{C}$ 1, basically, implies that given a path $\mathbf{a} \oplus\langle\mathrm{A}, \mathrm{C}\rangle$ there exists a lighter path to node $\mathrm{C}$ given by $\mathbf{a} \oplus\langle\mathrm{A}, \mathrm{B}, \mathrm{C}\rangle$, if and only if, the path $\mathbf{a} \oplus\langle\mathrm{A}, \mathrm{B}\rangle$ is lighter than $\mathbf{a} \oplus\langle\mathrm{A}, \mathrm{C}\rangle$. Condition $\mathrm{C} 2$ replaces node $\mathrm{C}$ by any complete path $\mathbf{c}$ satisfying $w(\mathbf{a} \oplus\langle\mathrm{A}, \mathrm{B}\rangle)) \preceq w\left(\mathbf{a} \oplus\left\langle\mathrm{A}, \mathbf{c}_{0, j}\right\rangle\right)$ for all $j$.

The next theorem states the sufficiency of conditions $\mathrm{C} 1$ and $\mathrm{C} 2$ for the optimality of Dijkstra's algorithm in a directed hypergraph.

Theorem 1: If a routing metric $(Q, \oplus, w, \preceq)$ satisfies conditions $\mathrm{C} 1$ and C2, then Dijkstra's algorithm finds the optimal path.

Proof: Given a hypergraph $\mathcal{H}(V, E)$, the set of nodes $\mathcal{R}=V$, the path weight function $w$, and the origin node of the path search o, Dijkstra's algorithm returns a set of paths $\mathbf{p}_{\mathrm{o}, \mathrm{r}}$, from node o to every other network node $\mathrm{r} \in \mathcal{R} / \mathrm{o}$, as well as the weights associated to those paths $l_{\mathrm{o}, \mathrm{r}}$. The pseudo code of Dijkstra's algorithm is shown in Algorithm 1. Let us denote as $\mathcal{R}^{(i)}, \mathbf{p}_{\mathrm{o}, \mathrm{r}}^{(i)}$ and $l_{\mathrm{o}, \mathrm{r}}^{(i)}$, respectively, the state of the set $\mathcal{R}$ $\left(\mathcal{R}^{(0)}=V\right)$, the paths $\mathbf{p}_{\mathrm{o}, \mathrm{r}}$ and the weights $l_{\mathrm{o}, \mathrm{r}}$ at the beginning of the $i$-th iteration. Suppose the initial iteration is $i=0$. Let $\mathbf{u}[i]$ be the node extracted from $\mathcal{R}^{(i)}$ in lines 6-7 at iteration $i$. We say that the path $\mathbf{p}_{\mathrm{o}, \mathbf{u}[i]}$ from node o to $\mathbf{u}[i]$ is found at iteration $i$, as it is no longer updated by the algorithm.

We prove this theorem in two steps. First, we show that for a routing metric satisfying condition $\mathrm{C} 1$ in Definition 3, Dijkstra's algorithm at iterations $i=\{1, \ldots,|V|-1\}$ finds the path $\mathbf{p}_{\mathrm{o}, \mathbf{u}[i]}$ from the origin o to node $\mathbf{u}[i]$ as $\mathbf{u}_{0, i}=$ $\left\langle\mathbf{u}_{0, i-1}, \mathbf{u}[i]\right\rangle$ where $\mathbf{u}_{0, i-1}$ is the path found at iteration $i-1$, $(\mathbf{u}[0]=0)$ and $\mathbf{u}[i]$ is the node that satisfies

$$
\mathbf{u}[i]=\arg \min _{\mathbf{r} \in \mathcal{R}^{(i)}} w\left(\left\langle\mathbf{u}_{0, i-1}, \mathbf{r}\right\rangle\right)
$$

where $\mathcal{R}^{(i)}=\mathcal{R}^{(i-1)} / \mathbf{u}_{0, i-1}$. Then, we show that for a routing metric satisfying also condition $\mathrm{C} 2$, the path $\mathbf{u}_{0, i}$ is the lightest path from node o to node $\mathbf{u}[i]$.
Step 1: Suppose condition $l_{\mathrm{o}, r} \succeq w_{\mathrm{u} \oplus \mathrm{r}}$ is satisfied for every node $\mathrm{r} \in \mathcal{R}^{(i)}$, from iteration 0 to $i$. At these iterations, the weights $l_{\mathrm{o}, \mathrm{r}}$ and paths $\mathbf{p}_{\mathrm{o}, \mathrm{r}}$ are updated $\forall \mathrm{r} \in \mathcal{R}^{(i)}$ in line 11 , as

$$
\begin{aligned}
\mathbf{p}_{\mathrm{o}, \mathrm{r}}^{(i+1)} & =\mathbf{p}_{\mathrm{o}, \mathbf{u}[i]}^{(i)} \oplus\langle\mathbf{u}[i], \mathbf{r}\rangle, \\
l_{\mathrm{o}, \mathrm{r}}^{(i+1)} & =w\left(\mathbf{p}_{\mathrm{o}, \mathrm{r}}^{(i+1)}\right) .
\end{aligned}
$$

At iteration 0 , line 6 of Dijkstra's algorithm selects $\mathbf{u}[0]=0$ and thus, the paths $\mathbf{p}_{\mathrm{o}, \mathrm{r}} \forall \mathrm{r} \in \mathcal{R}^{(0)}$ are updated, as

$$
\mathbf{p}_{\mathrm{o}, \mathrm{r}}^{(1)}=\langle\mathbf{u}[0], \mathrm{r}\rangle \text {. }
$$

At iteration 1 , line 6 selects $\mathbf{u}[1]$. Note that $\mathbf{p}_{\mathrm{o}, \mathbf{u}[1]}^{(1)}=$ $\langle\mathbf{u}[0], \mathbf{u}[1]\rangle$, and thus, the paths $\forall \mathbf{r} \in \mathcal{R}^{(1)}$ are updated as

$$
\mathbf{p}_{\mathrm{o}, \mathrm{r}}^{(2)}=\langle\mathbf{u}[0], \mathbf{u}[1], \mathbf{r}\rangle \text {. }
$$

Observe that at every iteration the paths $\mathbf{p}_{\mathrm{o}, \mathrm{r}}$ for every node $\mathrm{r}$, share the common root path $\mathbf{u}_{1, i}=\langle\mathbf{u}[1], \ldots, \mathbf{u}[i]\rangle$, i.e. $\mathbf{p}_{\mathrm{o}, \mathrm{r}}^{(i+1)}=\mathbf{u}_{0, i} \oplus\langle\mathbf{u}[i], \mathbf{r}\rangle=\left\langle\mathbf{u}_{0, i}, \mathrm{r}\right\rangle$ for all $\mathrm{r}$. Consequently, node $\mathbf{u}[i]$ in line 6 is chosen according to (8). Observe that

$$
\begin{aligned}
\mathbf{u}[i] & =\arg \min _{\mathbf{r} \in \mathcal{R}^{(i)}} l_{\mathrm{o}, \mathrm{r}}^{(i)}, \\
& =\arg \min _{\mathbf{r} \in \mathcal{R}^{(i)}} w\left(\mathbf{p}_{\mathrm{o}, \mathrm{r}}^{(i)}\right), \\
& =\arg \min _{\mathbf{r} \in \mathcal{R}^{(i)}} w\left(\left\langle\mathbf{u}_{0, i-1}, \mathbf{r}\right\rangle\right) .
\end{aligned}
$$

It only remains to show that condition $l_{\mathrm{o}, \mathrm{r}} \succeq w_{\mathrm{u} \oplus \mathrm{r}}$ in line 10 is always satisfied as previously assumed. We show that if condition $l_{\mathrm{o}, \mathrm{r}} \succeq w_{\mathrm{u} \oplus \mathrm{r}}$ is satisfied from iteration 0 to $i-1$, it is also satisfied at iteration $i$. At the beginning of iteration $i$, we have that $\mathbf{p}_{\mathrm{o}, \mathrm{r}}^{(i)}=\left\langle\mathbf{u}_{0, i-1}, \mathbf{r}\right\rangle$ and $l_{\mathrm{o}, \mathrm{r}}^{(i)}=w\left(\left\langle\mathbf{u}_{0, i-1}, \mathbf{r}\right\rangle\right)$. If node $\mathbf{u}[i]$ is chosen in line 6 , according to (8) it is satisfied that $w\left(\left\langle\mathbf{u}_{0, i-1}, \mathbf{u}[i]\right\rangle\right) \preceq w\left(\left\langle\mathbf{u}_{0, i-1}, \mathbf{r}\right\rangle\right)$ for all $\mathbf{r} \in \mathcal{R}^{(i)}$, then, in line 9 , we compute

$$
w_{\mathbf{u} \oplus \mathbf{r}}=w\left(\left\langle\mathbf{u}_{0, i-1}, \mathbf{u}[i], \mathbf{r}\right\rangle\right) .
$$

It is then a direct consequence of condition $\mathrm{C} 1$, that

$$
\begin{aligned}
w_{\mathbf{u} \oplus \mathbf{r}} & =w\left(\left\langle\mathbf{u}_{0, i-1}, \mathbf{u}[i], \mathbf{r}\right\rangle\right), \\
& \preceq w\left(\left\langle\mathbf{u}_{0, i-1}, \mathbf{r}\right\rangle\right), \\
& =l_{\mathrm{o}, \mathrm{r}}^{(i)}
\end{aligned}
$$

and thus, condition $l_{\mathrm{o}, \mathrm{r}} \succeq w_{\mathrm{u} \oplus \mathrm{r}}$ is also satisfied at the $i-$ th iteration for every node. Finally, notice that the condition $l_{\mathrm{o}, \mathrm{r}} \succeq$ $w_{\mathrm{u} \oplus \mathrm{r}}$ is trivially satisfied at $i=0$, since initially $l_{\mathrm{o}, \mathrm{r}}=\infty$ for all $\mathrm{r}$.

Step 2: Next, we show that if a routing metric satisfies conditions $\mathrm{C} 1$ and $\mathrm{C} 2$, then the path $\mathbf{u}_{0, i}$ is the lightest paths from node $\mathbf{u}[0]$ to node $\mathbf{u}[i]$. We prove this result by contradiction. Assume that the lightest path $\mathbf{s}$ between a given source-destination pair S-D satisfies $\mathbf{s}[i]=\mathbf{u}[i]$ for $j<i$ but $\mathbf{s}[i] \neq \mathbf{u}[i]$. Given that $\mathbf{u}[i]$ is choose according to (8), we have that

$$
w\left(\left\langle\mathbf{s}_{0, i-1}, \mathbf{u}[i]\right\rangle\right) \preceq w\left(\left\langle\mathbf{s}_{0, i-1}, \mathbf{s}[i]\right\rangle\right) .
$$

Let us denote $\mathrm{B}=\mathbf{u}[i]$. We define a new path from the source $\mathrm{S}$ to the destination $\mathrm{D}$ by including node $\mathrm{B}$ between nodes 
$\mathbf{s}[i-1]$ and $\mathbf{s}[i]$, namely $\left\langle\mathbf{s}_{0, i-1}, \mathbf{B}, \mathbf{s}_{i, L+1}\right\rangle$. We show next that this path is lighter than the original path $\mathbf{s}=\left\langle\mathbf{s}_{0, i-1}, \mathbf{s}_{i, L+1}\right\rangle$ which contradicts the assumption that $\mathbf{s}$ is the lightest path. Denote $\mathbf{a}=\mathbf{s}_{0, i-1}$, and $\mathbf{c}=\mathbf{s}_{i, L+1}$ with $\mathbf{c}[0]=\mathbf{s}[i]=\mathbf{C}$. In this case, the newly defined path reads $\langle\mathbf{a}, \mathrm{B}, \mathbf{c}\rangle$, and from condition (8), we have that

$$
w(\langle\mathbf{a}, \mathbf{B}\rangle) \preceq w(\langle\mathbf{a}, \mathbf{C}\rangle) .
$$

We consider the following two possible situations: $i)$ Assume that the partial path $\mathbf{c}$ satisfies $w(\langle\mathbf{a}, \mathbf{B}\rangle)) \preceq w\left(\left\langle\mathbf{a}, \mathbf{c}_{0, j}\right\rangle\right)$ for all $j$. In this case, it is a direct consequence of condition $\mathrm{C} 2$ that $w(\langle\mathbf{a}, \mathbf{B}, \mathbf{c}\rangle) \preceq w(\langle\mathbf{a}, \mathbf{c}\rangle)$ or, equivalently, that $w\left(\left\langle\mathbf{s}_{0, i-1}, \mathbf{B}, \mathbf{s}_{i, L+1}\right\rangle\right) \preceq w(\mathbf{s})$. ii $)$ Instead, assume that the partial path $\mathbf{c}$ satisfies

$$
w(\langle\mathbf{a}, \mathbf{B}\rangle)) \preceq w\left(\left\langle\mathbf{a}, \mathbf{c}_{0, j}\right\rangle\right),
$$

for all $j<m$ but not at $j=m$, this is

$$
w(\langle\mathbf{a}, \mathbf{B}\rangle) \succeq w\left(\left\langle\mathbf{a}, \mathbf{c}_{0, m-1}, \mathbf{D}\right\rangle\right)
$$

with $\mathbf{c}[m]=\mathrm{D}$. In this case, we show next, that conditions $\mathrm{C} 1$ and $\mathrm{C} 2$ require that $w(\langle\mathbf{a}, \mathrm{B}\rangle)) \succeq w(\langle\mathbf{a}, \mathrm{D}\rangle)$, which contradicts the assumption that node $\mathrm{B}$ is chosen in (8). To prove this result, we iteratively remove the node prefixed to node $\mathrm{D}$ in the path $\left\langle\mathbf{a}, \mathrm{B}, \mathbf{c}_{0, m-1}, \mathrm{D}\right\rangle$, until node $\mathrm{B}$ is finally removed. We show that a lighter path to node $\mathrm{D}$ is obtained at every iteration. We begin by removing node $\mathbf{c}[m-1]$. Denote, $\mathbf{c}[m-1]=\mathrm{C}$ and $\mathbf{a}^{\prime}=\left\langle\mathbf{a}, \mathrm{B}, \mathbf{c}_{0, m-2}\right\rangle$. Then, combining (17) for $j=m-1$, and (18), we have that

$$
\begin{aligned}
w\left(\left\langle\mathbf{a}^{\prime}, \mathbf{C}, \mathbf{D}\right\rangle\right) & \preceq w(\langle\mathbf{a}, \mathbf{B}\rangle), \\
& \preceq w\left(\left\langle\mathbf{a}^{\prime}, \mathbf{C}\right\rangle\right)
\end{aligned}
$$

and thus, satisfying conditions $\mathrm{C} 1$ implies that

$$
w\left(\left\langle\mathbf{a}^{\prime}, \mathbf{D}\right\rangle\right) \preceq w\left(\left\langle\mathbf{a}^{\prime}, \mathbf{C}, \mathbf{D}\right\rangle\right)
$$

or, equivalently,

$$
w\left(\left\langle\mathbf{a}, \mathbf{B}, \mathbf{c}_{0, m-2}, \mathrm{D}\right\rangle\right) \preceq w\left(\left\langle\mathbf{a}, \mathbf{B}, \mathbf{c}_{0, m-1}, \mathrm{D}\right\rangle\right) .
$$

This is, by removing node $\mathbf{c}[m-1]$, we obtain a lighter path to node D. Next, we remove node $\mathbf{c}[m-2]$. By combining (17) with (18) for $j=m-2$, we can write

$$
\begin{aligned}
w\left(\left\langle\mathbf{a}, \mathbf{B}, \mathbf{c}_{0, m-3}, \mathbf{c}[m-2]\right\rangle\right) & \succeq w\left(\left\langle\mathbf{a}, \mathbf{B}, \mathbf{c}_{0, m-1}, \mathbf{D}\right\rangle\right), \\
& \succeq w\left(\left\langle\mathbf{a}, \mathbf{B}, \mathbf{c}_{0, m-2}, \mathbf{D}\right\rangle\right) .
\end{aligned}
$$

Now, denote $\mathbf{C}=\mathbf{c}[m-2]$ and $\mathbf{a}^{\prime}=\left\langle\mathbf{a}, \mathbf{B}, \mathbf{c}_{0, m-3}\right\rangle$, then (22b) can be rewritten as

$$
w\left(\left\langle\mathbf{a}^{\prime}, \mathbf{C}, \mathbf{D}\right\rangle\right) \preceq w\left(\left\langle\mathbf{a}^{\prime}, \mathbf{C}\right\rangle\right)
$$

and thus, following previous arguments, satisfying condition C1 implies

$$
\begin{aligned}
w\left(\left\langle\mathbf{a}, \mathrm{B}, \mathbf{c}_{0, m-3}, \mathrm{D}\right\rangle\right) & \preceq w\left(\left\langle\mathbf{a}, \mathbf{B}, \mathbf{c}_{0, m-2}, \mathrm{D}\right\rangle\right), \\
& \preceq w\left(\left\langle\mathbf{a}, \mathbf{B}, \mathbf{c}_{0, m-1}, \mathrm{D}\right\rangle\right) .
\end{aligned}
$$

This is, by removing node $\mathbf{c}[m-2]$, we obtain a lighter path to node $\mathrm{D}$. We repeat this procedure until we remove node $\mathrm{B}$.

\section{Optimality Analysis of Minimum Energy ACCumulative Routing Metrics}

In this section, we study the optimality of Dijkstra's algorithm for the minimum energy accumulative path weight functions presented in Section III.

\section{A. Decode-and-Forward Relaying}

We begin by discussing the optimality of Dijkstra's algorithm for the TM path weight function in (1). In this case, the routing problem can be modeled using a graph, where the metric of the edge between nodes $u$ and $v$ is given by $\beta\left(e_{\mathrm{u}, \mathrm{v}}\right)=g_{\mathrm{u}, \mathrm{v}}$ and thus, in that case, right-monotonicity and right-isotonicity are not only sufficient but also necessary conditions.

Let us consider any path weight function that admits the following recursive computation in a graph

$w\left(\mathbf{p}_{0, i}\right)= \begin{cases}\frac{1}{\beta_{\mathbf{p}[i-1], \mathbf{p}[i]}}+w\left(\mathbf{p}_{0, i-1}\right), & \text { if } w\left(\mathbf{p}_{0, i}\right) \geq w\left(\mathbf{p}_{0, i-1}\right) \\ \infty & \text { otherwise }\end{cases}$

where $\beta_{\mathrm{u}, \mathrm{v}}$ is the metric associated to the edge $e_{\mathrm{u}, \mathrm{v}}$.

We can obtain the path weight in (1) for the TM, from (25) by setting $\beta\left(e_{\mathrm{u}, \mathrm{v}}\right)=\beta_{\mathrm{u}, \mathrm{v}}=g_{\mathrm{u}, \mathrm{v}}$ for all $\mathrm{u}, \mathrm{v}$. Given that $g_{\mathrm{u}, \mathrm{v}} \geq 0$ for all $\mathrm{u}$ and $\mathrm{v}$, it is also satisfied that $w\left(\mathbf{p}_{0, i}\right) \geq w\left(\mathbf{p}_{0, i-1}\right)$ for all $i$.

Right-monotonicity is implicit in the path weight definition (25) as we require $w\left(\mathbf{p}_{0, i}\right) \geq w\left(\mathbf{p}_{0, i-1}\right)$. Notice that it is satisfied even if $\beta_{\mathbf{p}[i-1], \mathbf{p}[i]}<0$. To show right-isotonicity, consider the paths $\mathbf{a}$ and $\mathbf{b}$ between nodes $\mathrm{A}$ and $\mathrm{B}$, with $w(\mathbf{a})$ and $w(\mathbf{b})$ satisfying $w(\mathbf{a}) \leq w(\mathbf{b})$. Let us concatenate, node $\mathrm{C}$ to the right of $\mathbf{a}$ and $\mathbf{b}$, then the path weight at node $\mathrm{C}$ is given by

$$
\begin{aligned}
& w(\mathbf{a} \oplus\langle\mathrm{B}, \mathrm{C}\rangle)=\frac{1}{\beta_{\mathrm{B}, \mathrm{C}}}+w(\mathbf{a} \oplus \mathbf{C}), \\
& w(\mathbf{b} \oplus\langle\mathrm{B}, \mathrm{C}\rangle)=\frac{1}{\beta_{\mathrm{B}, \mathrm{C}}}+w(\mathbf{b} \oplus \mathbf{C}) .
\end{aligned}
$$

If $\beta_{\mathrm{B}, \mathrm{C}} \geq 0$ then $w(\mathbf{a}) \leq w(\mathbf{b})$ implies $w(\mathbf{a} \oplus\langle\mathrm{B}, \mathrm{C}\rangle)<$ $w(\mathbf{b} \oplus\langle\mathbf{B}, \mathbf{C}\rangle)$. Instead if $\beta_{\mathrm{B}, \mathrm{C}}<0$, then $w(\mathbf{a} \oplus\langle\mathbf{B}, \mathbf{C}\rangle)=$ $w(\mathbf{b} \oplus\langle\mathbf{B}, \mathbf{C}\rangle)$.

The path weight in (3) for the decode-and-forward relaying in AM networks can only be defined in a hypergraph. It is well-know that the problem of finding the optimal path for this routing metric is NP-complete [3] and [4]. Accordingly, although this path weight satisfies right-monotonicity, it does not satisfies right-isotonicity, nor conditions $\mathrm{C} 1$ and $\mathrm{C} 2$, and thus, the optimality of Dijkstra's algorithm can not be guaranteed.

Let us instead discuss the optimality of Dijkstra's algorithm for a simpler AM with DF relaying. Let us limit the accumulative capabilities at nodes and suppose that every relay node only listens to the source, and to the immediately previous node. Then, the power received at node $\mathbf{p}[i]$ from nodes $0<j<i-1$ is not accumulated. Substituting $g_{\mathbf{p}[j], \mathbf{p}[i]}=0$ for $0<j<i-1$ into (3), we obtain the path weight function 
for this situation as

$$
\begin{aligned}
w\left(\mathbf{p}_{0, i}\right) & =\frac{1}{g_{i-1, i}}+\frac{g_{i-1, i} w\left(\mathbf{p}_{0, i-1}\right)-g_{0, i} w\left(\mathbf{p}_{0,1}\right)}{g_{i-1, i}} \\
& =\frac{g_{0,1}-g_{0, i}}{g_{i-1, i} g_{0,1}}+w\left(\mathbf{p}_{0, i-1}\right)
\end{aligned}
$$

if $w\left(\mathbf{p}_{0, i}\right)>w\left(\mathbf{p}_{0, i-1}\right)$ and $w\left(\mathbf{p}_{0, i}\right)=\infty$, otherwise. This path weight function can now be defined using a graph. Suppose, the first relay is fixed, then, we could define the metric associated to the edge between nodes $u$, and $v$, as

$$
\beta\left(e_{\mathrm{u}, \mathrm{v}}\right)=\beta_{\mathrm{u}, \mathrm{v}}=\frac{g_{\mathrm{u}, \mathrm{v}} g_{\mathrm{S}, \mathrm{R}}}{g_{\mathrm{S}, \mathrm{R}}-g_{\mathrm{S}, \mathrm{v}}}
$$

and write (27) as in (25). Now, we can find the optimal path from a source-relay pair $\langle\mathrm{S}, \mathrm{R}\rangle$ to every other network node using Dijkstra's algorithm. We however need to repeat the search for all possible first relay nodes in order to get the optimal path from the source node.

\section{B. Cut-set bound}

Next, we study the optimality of Dijkstra's algorithm for the cut-set bound path weight function in (5). This path weight function can only be computed over a hypergraph, it does not satisfies right-monotonicity nor right-isotonicity. However, as we show in next theorem it satisfies conditions $\mathrm{C} 1$ and $\mathrm{C} 2$, and thus, the optimal path can be found using Dijkstra's algorithm.

Theorem 2: Consider a hypergraph $\mathcal{H}(V, E)$ with only one edge per node, where the metric associated to the hyperedge of node $\mathrm{u}$ is defined as $\beta\left(e_{\mathrm{u}}\right)=\left\{g_{\mathrm{u}, \mathrm{v}}, \forall \mathrm{v}\right\}$. The cut-set path weight function in (5) satisfies conditions $\mathrm{C} 1$ and $\mathrm{C} 2$, and thus, Dijkstra's algorithm finds the optimal path.

Proof: We first show that condition $\mathrm{C} 1$ is satisfied. Let $\mathbf{a}$ be a path with $L_{a}$ relays nodes, $\mathbf{a}=\left\langle\mathbf{a}[0], \ldots, \mathbf{a}\left[L_{a}+1\right]\right\rangle$. Given the paths $\langle\mathbf{a}, \mathrm{X}\rangle$, where $\mathrm{X}$ is any network node $\mathrm{X} \in V$, define

$$
\begin{aligned}
& N_{\mathrm{X}}=1+\sum_{l=1}^{L_{\mathbf{a}}+1} g_{\mathbf{a}[l], \mathrm{X}} w\left(\mathbf{a}_{0, j}\right) \\
& D_{\mathrm{X}}=\sum_{l=0}^{L_{a}+1} g_{\mathbf{a}[l], \mathrm{X}} .
\end{aligned}
$$

Then, the weight of the paths evaluated in condition $\mathrm{C} 1$ are given by

$$
\begin{aligned}
w(\langle\mathbf{a}, \mathrm{C}\rangle) & =\frac{N_{\mathrm{C}}}{D_{\mathrm{C}}}, \\
w(\langle\mathbf{a}, \mathbf{B}\rangle) & =\frac{N_{\mathrm{B}}}{D_{\mathrm{B}}}, \\
w(\langle\mathbf{a}, \mathbf{B}, \mathbf{C}\rangle) & =\frac{N_{\mathrm{C}}+g_{\mathrm{B}, \mathrm{C}} w(\langle\mathbf{a}, \mathrm{B}\rangle)}{D_{\mathrm{C}}+g_{\mathrm{B}, \mathrm{C}}}, \\
w(\langle\mathbf{a}, \mathbf{C}, \mathbf{B}\rangle) & =\frac{N_{\mathrm{B}}+g_{\mathrm{C}, \mathrm{B}} w(\langle\mathbf{a}, \mathrm{C}\rangle)}{D_{\mathrm{B}}+g_{\mathrm{C}, \mathrm{B}}} .
\end{aligned}
$$

Suppose that $w(\langle\mathbf{a}, \mathrm{B}\rangle) \preceq w(\langle\mathbf{a}, \mathbf{C}\rangle)$, and $g_{\mathrm{B}, \mathrm{C}}, g_{\mathrm{C}, \mathrm{B}} \geq 0$, then ${ }^{7}$ observe that

$$
\begin{aligned}
w(\langle\mathbf{a}, \mathrm{B}, \mathrm{C}\rangle) & =\frac{N_{\mathrm{C}}+g_{\mathrm{B}, \mathrm{C}} w(\langle\mathbf{a}, \mathrm{B}\rangle)}{D_{\mathrm{C}}+g_{\mathrm{B}, \mathrm{C}}}, \\
& \preceq \frac{N_{\mathrm{C}}+g_{\mathrm{B}, \mathrm{C}} w(\langle\mathbf{a}, \mathrm{C}\rangle)}{D_{\mathrm{C}}+g_{\mathrm{B}, \mathrm{C}}}, \\
& =w(\langle\mathbf{a}, \mathbf{C}\rangle)
\end{aligned}
$$

and

$$
\begin{aligned}
w(\langle\mathbf{a}, \mathbf{C}, \mathrm{B}\rangle) & =\frac{N_{\mathrm{B}}+g_{\mathrm{C}, \mathrm{B}} w(\langle\mathbf{a}, \mathrm{C}\rangle)}{D_{\mathrm{B}}+g_{\mathrm{C}, \mathrm{B}}}, \\
& \succeq \frac{N_{\mathrm{B}}+g_{\mathrm{C}, \mathrm{B}} w(\langle\mathbf{a}, \mathrm{B}\rangle)}{D_{\mathrm{B}}+g_{\mathrm{C}, \mathrm{B}}}, \\
& =w(\langle\mathbf{a}, \mathrm{B}\rangle) .
\end{aligned}
$$

By isolating $w(\langle\mathbf{a}, \mathbf{B}\rangle)$ from (30) and using (32), we observe that

$$
\begin{aligned}
w(\langle\mathbf{a}, \mathrm{B}\rangle) & =w(\langle\mathbf{a}, \mathbf{B}, \mathbf{C}\rangle)+\frac{D_{\mathrm{C}} w(\langle\mathbf{a}, \mathbf{B}, \mathbf{C}\rangle)-N_{\mathbf{C}}}{g_{\mathrm{B}, \mathrm{C}}}, \\
& \preceq w(\langle\mathbf{a}, \mathbf{B}, \mathbf{C}\rangle)+\frac{D_{\mathrm{C}} w(\langle\mathbf{a}, \mathbf{C}\rangle)-N_{\mathrm{C}}}{g_{\mathrm{B}, \mathrm{C}}} \\
& =w(\langle\mathbf{a}, \mathbf{B}, \mathbf{C}\rangle) .
\end{aligned}
$$

Notice that, the reverse is also true, i.e. satisfying (32) implies

$$
\begin{aligned}
w(\langle\mathbf{a}, \mathrm{B}\rangle) & =\frac{w(\langle\mathbf{a}, \mathbf{B}, \mathbf{C}\rangle)\left(D_{\mathrm{C}}+g_{\mathrm{B}, \mathrm{C}}\right)-N_{\mathrm{C}}}{g_{\mathrm{B}, \mathrm{C}}}, \\
& \preceq \frac{w(\langle\mathbf{a}, \mathrm{C}\rangle)\left(D_{\mathrm{C}}+g_{\mathrm{B}, \mathrm{C}}\right)-N_{\mathrm{C}}}{g_{\mathrm{B}, \mathrm{C}}}, \\
& =w(\langle\mathbf{a}, \mathbf{C}\rangle) .
\end{aligned}
$$

Next, by isolating $w(\langle\mathbf{a}, \mathbf{C}\rangle)$ from (31) and using (33), we observe that

$$
\begin{aligned}
w(\langle\mathbf{a}, \mathbf{C}\rangle) & =w(\langle\mathbf{a}, \mathbf{C}, \mathbf{B}\rangle)+\frac{w\langle\mathbf{a}, \mathbf{C}, \mathbf{B}\rangle) D_{\mathrm{B}}-N_{\mathrm{B}}}{g_{\mathrm{C}, \mathrm{B}}}, \\
& \succeq w(\langle\mathbf{a}, \mathbf{C}, \mathbf{B}\rangle)+\frac{w(\langle\mathbf{a}, \mathbf{B}\rangle) D_{\mathrm{B}}-N_{\mathrm{B}}}{g_{\mathrm{C}, \mathrm{B}}}, \\
& =w(\langle\mathbf{a}, \mathbf{C}, \mathbf{B}\rangle) .
\end{aligned}
$$

Next, we show that condition $\mathrm{C} 2$ is also satisfied. Let us consider the paths $\langle\mathbf{a}, \mathbf{c}\rangle$ and $\langle\mathbf{a}, \mathrm{B}, \mathbf{c}\rangle$ where $\mathbf{c}=$ $\left\langle\mathbf{c}[0], \ldots, \mathbf{c}\left[L_{\mathbf{c}}+1\right]\right\rangle$ and denote the weight of the paths $\left\langle\mathbf{a}, \mathbf{c}_{0, i}\right\rangle$ as

$$
w\left(\left\langle\mathbf{a}, \mathbf{c}_{0, i}\right\rangle\right)=\frac{N_{i}}{D_{i}}
$$

for $i=0, \ldots, L_{\mathbf{c}}+1$. Let us decompose $N_{i}$ and $D_{i}$ as $N_{i}=$ $N_{\mathbf{a}, i}+N_{\mathbf{c}, i}$ and $D_{i}=D_{\mathbf{a}, i}+D_{\mathbf{c}, i}$ where

$$
\begin{aligned}
& N_{\mathbf{a}, i}=1+\sum_{l=0}^{L_{\mathbf{a}}+1} g_{\mathbf{a}[l], \mathbf{c}[i]} w\left(\mathbf{a}_{0, l}\right), D_{\mathbf{a}, i}=\sum_{l=0}^{L_{a}+1} g_{\mathbf{a}[l], \mathbf{c}[i]}, \\
& N_{\mathbf{c}, i}=\sum_{l=0}^{i-1} g_{\mathbf{c}[l], \mathbf{c}[i]} w\left(\left\langle\mathbf{a}, \mathbf{c}_{0, l}\right\rangle\right), D_{\mathbf{c}, i}=\sum_{l=0}^{i-1} g_{\mathbf{a}[l], \mathbf{c}[i]} .
\end{aligned}
$$


Then, observe that if $w(\langle\mathbf{a}, \mathbf{B}\rangle) \preceq w\left(\left\langle\mathbf{a}, \mathbf{c}_{0, l}\right\rangle\right)$ for all $l$ and $w\left(\left\langle\mathbf{a}, \mathbf{B}, \mathbf{c}_{0, l}\right\rangle\right) \preceq w\left(\left\langle\mathbf{a}, \mathbf{c}_{0, l}\right\rangle\right)$ for $l=1$ to $l=i-1$, then $w\left(\left\langle\mathbf{a}, \mathbf{B}, \mathbf{c}_{0, i}\right\rangle\right)$

$$
\begin{aligned}
& =\frac{N_{\mathbf{a}, i}+g_{\mathrm{B}, \mathbf{c}[i]} w(\langle\mathbf{a}, \mathbf{B}\rangle)+\sum_{l=0}^{i-1} g_{\mathbf{c}[l], \mathbf{c}[i]} w\left(\left\langle\mathbf{a}, \mathbf{B}, \mathbf{c}_{0, l}\right\rangle\right)}{D_{\mathbf{a}, i}+g_{\mathrm{B}, \mathbf{c}[i]}+D_{\mathbf{c}, i}} \\
& \preceq \frac{N_{i}+g_{\mathrm{B}, \mathbf{c}[i]} w(\langle\mathbf{a}, \mathbf{B}\rangle)}{D_{i}+g_{\mathrm{B}, \mathbf{c}[i]}} \\
& =\frac{N_{i}+g_{\mathrm{B}, \mathbf{c}[i]} \frac{w(\langle\mathbf{a}, \mathrm{B}\rangle)}{w\left(\mathbf{a}, \mathbf{c}_{0, i}\right)} \frac{N_{i}}{D_{i}}}{D_{i}+g_{\mathrm{B}, \mathbf{c}[i]}} \\
& \preceq \frac{N_{i}+g_{\mathrm{B}, \mathbf{c}[i] \frac{N_{i}}{D_{i}}}}{D_{i}+g_{\mathrm{B}, \mathbf{c}[i]}} \\
& =\frac{N_{i}}{D_{i}}=w\left(\left\langle\mathbf{a}, \mathbf{c}_{0, i}\right\rangle\right)
\end{aligned}
$$

where inequality (38b) is due to $w\left(\left\langle\mathbf{a}, \mathbf{B}, \mathbf{c}_{0, l}\right\rangle\right) \preceq$ $w\left(\left\langle\mathbf{a}, \mathbf{c}_{0, l}\right\rangle\right)$ for $l=0, \ldots, i-1$ and inequality (38d) is due to $w(\langle\mathbf{a}, \mathbf{B}\rangle) \preceq w\left(\mathbf{a}, \mathbf{c}_{0, i}\right)$.

\section{CONCLUSIONS}

In this paper, we studied the routing problem in accumulative multi-hop networks. We showed that as opposed to traditional multi-hoping where the network is well modeled by graph, for routing in accumulative networks, the network needs to be modeled by a hypergraph. We studied the properties that guarantee that Dijkstra's algorithm finds the optimal path in such networks, and presented sufficient conditions for the optimality. These conditions are particularized for the minimum energy routing problem with decode-and-forward relays and for the cut-set bound.

\section{REFERENCES}

[1] J. Sobrinho, "An algebraic theory of dynamic network routing," Networking, IEEE/ACM Transactions on, vol. 13, no. 5, pp. 1160 - 1173, oct. 2005.

[2] Y. Yang and J. Wang, "Design guidelines for routing metrics in multihop wireless networks," in IEEE INFOCOM, 2008, pp. $1615-1623$.

[3] I. Maric and R. Yates, "Cooperative multihop broadcast for wireless networks," IEEE J. Select. Areas Commun., vol. 22, no. 6, pp. 10801088, Aug. 2004.

[4] J. Chen, L. Jia, X. Liu, G. Noubir, and R. Sundaram, "Minimum energy accumulative routing in wireless networks," in Proc. IEEE INFOCOM, vol. 3, Mar. 2005, pp. 1875 - 1886.

[5] A. Molisch, N. Mehta, J. Yedidia, and J. Zhang, "Cooperative relay networks using fountain codes," in Proc. IEEE Global Communications Conference (GLOBECOM), Nov. 2006.

[6] J. Castura and Y. Mao, "Rateless coding over fading channels," Communications Letters, IEEE, vol. 10, no. 1, pp. 46-48, Jan 2006.

[7] S. C. Draper, L. Liu, A. F. Molisch, and J. S.Yedidia, "Cooperative routing for wireless networks using mutual-information accumulation," IEEE Trans. Inform. Theory, vol. 57, Aug. 2011.

[8] Z. Yang and A. Høst-Madsen, "Routing and power allocation in asynchronous gaussian multiple-relay channels," EURASIP Journal on Wireless Communications and Networking, 2006.

[9] T. Girici and A. C. Kazez, "Energy efficient routing with mutual information accumulation," in Modeling and Optimization in Mobile, Ad Hoc and Wireless Networks (WiOpt), 2012 10th International Symposium on, may 2012, pp. $425-430$.
[10] R. Yim, N. Mehta, A. F. Molisch, and J. Zhang, "Progressive accumulative routing in wireless networks," in Proc. IEEE Global Communications Conference (GLOBECOM), Nov. 2006.

[11] T. Cover and J. A. Thomas, Elements of Information Theory, New York: Wiley, 1991.

[12] "Linear connectivity problems in directed hypergraphs," Theoretical Computer Science, vol. 410.

[13] R. Urgaonkar and M. Neely, "Optimal routing with mutual information accumulation in wireless networks," Selected Areas in Communications, IEEE Journal on, vol. 30, no. 9, pp. 1730 -1737, october 2012.

[14] A. Avestimehr, S. Diggavi, and D. Tse, "Wireless network information flow: A deterministic approach," Information Theory, IEEE Transactions on, vol. 57, no. 4, pp. 1872-1905, 2011.

:

